\title{
The protozoan Toxoplasma gondii: neurotoxicological relevance beyond the typical clinical pictures
}

\author{
Klaus Golka • Michael Falkenstein • \\ Patrick D. Gajewski
}

Received: 7 January 2015 / Accepted: 12 January 2015 / Published online: 25 January 2015

(C) Springer-Verlag Berlin Heidelberg 2015

The protozoan Toxoplasma gondii is a parasite, which is widespread worldwide. The prevalence of the infection in the general population is increasing with age and may vary considerably from country to country. Almost one-third of world's population is infected with $T$. gondii (Tenter et al. 2000). In Germany, the prevalence of the T. gondii-positive antibody status in the general population is estimated about $50 \%$, increasing with age (RKI 2009).

For decades, physicians feared the central nervous system complications of a first infection of the pregnant women, frequently leading to severe sequelae of the central nervous system of the newborn, and the toxoplasmosis of the eye. In immunocompetent individuals, the symptoms of an acute infection are generally mild and uncharacteristic. They may resemble a mild flu, with symptoms like lymphadenopathy, low grade fever, generalized malaise, mild to extreme tiredness and muscle pain (ACMSF 2012). However, the latent infection may become highly dangerous when the immune system is impaired, e.g., by HIV/ AIDS or by chronic alcoholism. It is estimated that up to $30 \%$ of patients diagnosed with AIDS will get a T. gondii encephalitis. Furthermore, people with suppressed immune system, e.g., after organ transplantation, have a clearly higher risk to contract a toxoplasmosis also by inhalation of the protozoan, e.g., via contaminated soil.

K. Golka $(\bowtie) \cdot$ M. Falkenstein · P. D. Gajewski

Leibniz Research Institute for Working Environment and Human

Factors (IfADo), Ardeystr. 67, 44139 Dortmund, Germany

e-mail: golka@ifado.de

M. Falkenstein

e-mail: falkenstein@ifado.de

P. D. Gajewski

e-mail: gajewski@ifado.de
In the past, a considerable number of papers were published, which reported an association of different negative effects in persons with a latent $T$. gondii infection (Flegr et al. 2014), particularly on the central nervous system (Dalimi and Abdoli 2012; Webster et al. 2013).

Flegr et al. (2002) reported that $T$. gondii-positive seroprevalence in people involved in traffic accidents was higher than in the general population. Furthermore, they observed an even higher risk in people with higher or very high antibody levels. The authors stated that from literature, there was no indication for prolonged reaction times of $T$. gondii-infected persons. Therefore, they were in favor of an increased risk due to their impaired psychomotor performance, particularly a decrease in concentration. GalvánRamírez Mde et al. (2003) observed higher T. gondii antibody levels in drivers with traffic accidents than in controls. The question whether the observed finding was possibly due to a more aggressive traffic behavior was not discussed by the authors of the two papers. These two papers indicate a dose-effect relationship within the group of patients with a latent $T$. gondii infection.

Pedersen et al. (2012) investigated in a nation-wide register-based study on pregnant women in Denmark the potential risk of $T$. gondii infection regarding suicide. The authors observed an elevated rate of suicide attempts in T. gondii IgG-positive mothers and an even higher rate of suicides, compared to non-infected mothers. The authors interpreted the outcome of the study as due to an increased risk of self-aggressiveness.

Gajewski et al. (2014) reported an impaired working memory in individuals 65 years and older with a $T$. gondii IgG antibody level >50 IU/ml, compared to noninfected individuals $(\mathrm{IgG}=0 \mathrm{IU} / \mathrm{ml})$. Moreover, lower performance in $T$. gondii-positive seniors was found in a verbal memory test, both regarding immediate and 
delayed recognition and in recall from long-term memory assessed by the word fluency tests. The challenging observation was subject of several studies. Dickerson et al. (2014) investigated the association of $T$. gondii infection and cognitive functioning in persons with schizophrenia or bipolar disorders aged 18-65 years, compared to persons without psychiatric disorders aged 20-60 years. As initial analyses indicated significant associations with $T$. gondii IgM antibodies but not with T. gondii IgG antibodies, the authors focused on the association of $T$. gondii IgM antibody level and cognitive effects. The authors observed significant association between $T$. gondii IgM antibody level and cognitive parameters within the groups of individuals with bipolar disorder or no psychiatric disorder, but not within the schizophrenia group. The impact of IgM antibodies but not of $\mathrm{IgG}$ antibodies is surprising. In general, IgM antibodies arise from acute infections. The authors refer to a study of Robert-Gangneux et al. (2009), which reported that some individuals have been found to have persistent elevations of IgM antibodies in the absence of increased IgG antibodies or other serological evidence of recent $T$. gondii infection. They remark that the immunological mechanisms defining the persistence of IgM antibodies have not been elucidated. Mendy et al. (2014) investigated the impact of latent $T$. gondii infection on the memory in 4,485 participants of the Third National Health and Nutrition examination Survey (NHANES) aged 60 years and older. The authors observed an impairment of immediate but not of delayed memory, particularly in non-Hispanic Whites. In contrast, a study on 4,178 participants of NHANES aged 20-59 years demonstrated no association between latent toxoplasmosis and cognitive outcomes (Gale et al. 2014). Furthermore, an effect of the T. gondii infection on memory was observed in an experimental study in rats (Daniels et al. 2015). However, it should be mentioned that the first reports of a memory impairment due to $T$. gondii in infected rats and mice were published decades ago (Witting 1979; Piekarski 1981).

Furthermore, a considerable number of studies investigated a possible association between $T$. gondii and diseases of the central nervous system in humans, particularly schizophrenia. In a meta-analysis focusing on an association of infective agents and schizophrenia, Arias et al. (2012) included 8 studies investigating T. gondii infection markers in people diseased with schizophrenia and healthy controls. The authors stated that schizophrenia was 2.7 times more frequent in marker-positive persons than in marker-negative persons. These figures are in line with a previously published meta-analysis on the association of $T$. gondii and schizophrenia, based on 23 included studies, showing an odds ratio of 2.73 (Torrey et al. 2007).
In essence, recent publications indicate that the latent T. gondii infection in humans has some negative adverse effects on human health, which are, at least in some studies, associated with the antibody level. Thus, further studies on the effects of this protozoan on humans as well as further efforts to reduce the portion of $T$. gondii-positive persons in the general population are needed.

\section{References}

ACMSF Advisory Committee on the Microbiological Safety of Food (ACMSF) Ad Hoc Group on Vulnerable Groups (2012) Risk profile in relation to toxoplasma in the food chain. Food Standards Agency, 2012. http://www.food.gov.uk/sites/default/files/multimedia/pdfs/committee/acmsfrtaxopasm.pd. Accessed Dec 4, 2014

Arias I, Sorlozano A, Villegas E et al (2012) Infectious agents associated with schizophrenia: a meta-analysis. Schizophr Res 136:128-136

Dalimi A, Abdoli A (2012) Latent toxoplasmosis and human. Iran J Parasitol 7:1-17

Daniels BP, Sestito SR, Rouse ST (2015) An expanded task battery in the Morris water maze reveals effects of Toxoplasma gondii infection on learning and memory in rats. Parasitol Int 64:5-12

Dickerson F, Stallings C, Origoni A et al (2014) Antibodies to Toxoplasma gondii and cognitive functioning in schizophrenia, bipolar disorder, and nonpsychiatric controls. J Nerv Ment Dis 202:589-593

Flegr J, Havlícek J, Kodym P et al (2002) Increased risk of traffic accidents in subjects with latent toxoplasmosis: a retrospective case-control study. BMC Infect Dis 2:11

Flegr J, Prandota J, Sovičková M, Israili ZH (2014) Toxoplasmosisa global threat. Correlation of latent toxoplasmosis with specific disease burden in a set of 88 countries. PLoS One 9(3):e90203

Gajewski PD, Falkenstein M, Hengstler JG, Golka K (2014) Toxoplasma gondii impairs memory in infected seniors. Brain Behav Immun 36:193-199

Gale SD, Brown BL, Erickson LD, Berrett A, Hedges DW (2014) Association between latent toxoplasmosis and cognition in adults: a cross-sectional study. Parasitology 7:1-9

Galván-Ramírez Mde L, Sánchez-Orozco LV, Rodríguez LR (2013) Seroepidemiology of Toxoplasma gondii infection in drivers involved in road traffic accidents in the metropolitan area of Guadalajara, Jalisco, Mexico. Parasit Vectors 6:294

Mendy A, Vieira ER, Albatineh AN, Gasana J (2014) Immediate rather than delayed memory impairment in older adults with latent toxoplasmosis. Brain Behav Immun. doi:10.1016/j. bbi.2014.12.006

Pedersen MG, Mortensen PB, Norgaard-Pedersen B, Postolache TT (2012) Toxoplasma gondii infection and self-directed violence in mothers. Arch Gen Psychiatry 69:1123-1130

Piekarski G (1981) Behavioral alterations caused by parasitic infection in case of latent toxoplasma infection. Zentralbl Bakteriol Mikrobiol Hyg [A] 250:403-406

RKI (2009) Toxoplasmose RKI-Ratgeber für Ärzte. http://www.rki. de/DE/Content/Infekt/EpidBull/Merkblaetter/Ratgeber_Toxoplasmose.html;jsessionid=E0A60ABAC6C467D2435537A1D99 BB9A9.2_cid372. Accessed Dec 4, 2014

Robert-Gangneux F, Bourhis C, Chevrier S, Gangneux JP (2009) Evaluation of DPC immulite 2000 toxoplasma quantitative $\mathrm{IgG} /$ IgM kits for automated toxoplasmosis serology with immulite 2000. J Clin Lab Anal 23:336-340 
Tenter AM, Heckeroth AR, Weiss L (2000) Toxoplasma gondii: from animals to humans. Int J Parasitol 30:1217-1258

Torrey EF, Bartko JJ, Lun ZR, Yolken RH (2007) Antibodies to Toxoplasma gondii in patients with schizophrenia: a meta-analysis. Schizophr Bull 33:729-736

Webster JP, Kaushik M, Bristow GC, McConkey GA (2013) Toxoplasma gondii infection, from predation to schizophrenia: can animal behavior help us understand human behavior? J Exp Biol 216:99-112

Witting PA (1979) Learning capacity and memory of normal and Toxoplasma-infected laboratory rats and mice. Z Parasitenkd 61:29-51 